\title{
Application of Systems for Complex Integrative Automatic Assembly of Electronic Components Super Systems and Subsystems with Pre-Stamping of Incoming Parts and Elements
}

\author{
Dmitrii Shekhovtsov
}

\begin{abstract}
An essential part of any production process is to actively monitor the parameters of these processes in real time. The most promising method of active real-time monitoring is non-contact monitoring or monitoring in the mode of electromagnetic resonance spectroscopy. Since these methods are fundamentally new and are only being implemented in technological processes, it makes sense to consider them in more detail.

Keywords: non-contact monitoring, electromagnetic resonance spectroscopy
\end{abstract}

В современном производстве изготовление качественных компонентов систем оборудования является залогом финального качества надсистемы.

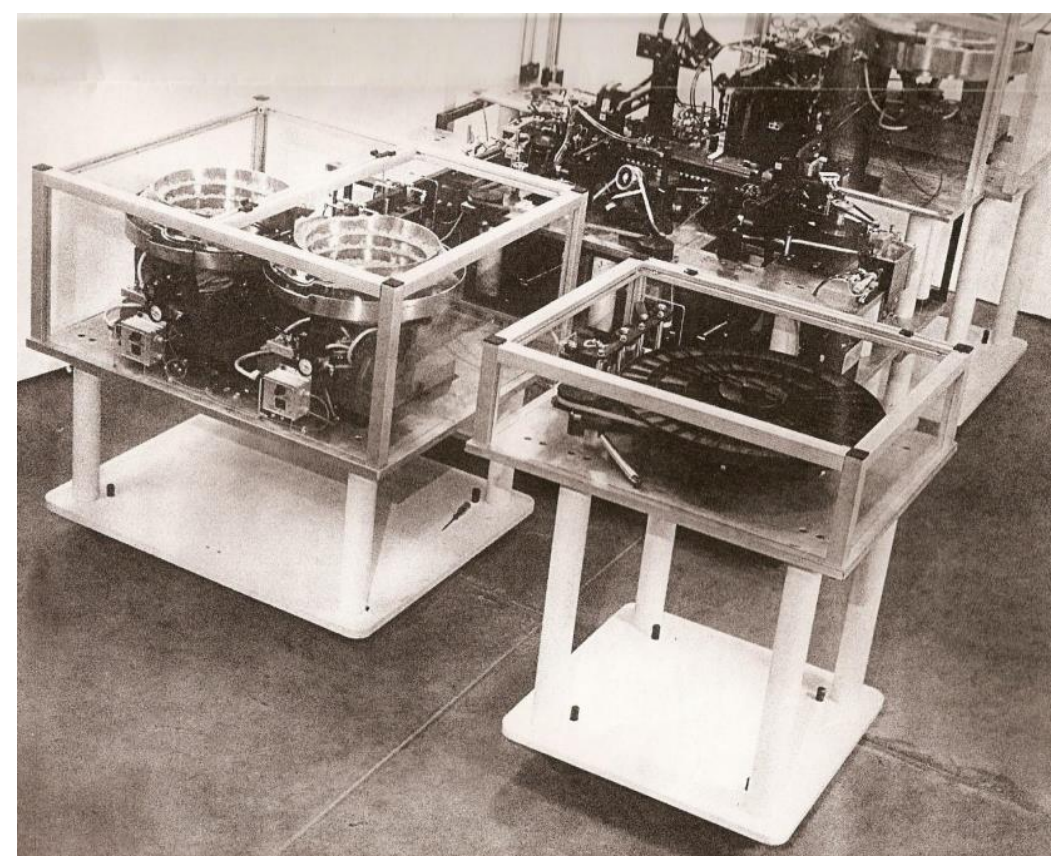

Рисунок 1. Автоматический производственный комплекс для изготовления, сборки и испытаний (контроля качества и работоспособности) контактных систем связи

Исходные технические требования контактных систем требуют, чтобы практически размерные параметры узлов и деталей контактных систем не отличались.

Такое требование заставляет применить соответствующий метод производства и сборки контактных систем, то есть штамповку входящих деталей (все размеры на всех штампованных деталях полностью идентичны) и передачу штампованных деталей на конвейер сборки автоматического производственного комплекса. 
Автоматический производственный комплекс построен таким образом, что штамповка деталей, включая пружинные контакты, ведётся на автономных установках, связанных конвейерами с центральным конвейером сборки и контроля (находится в центре производственной системы, показанной на рисунке).

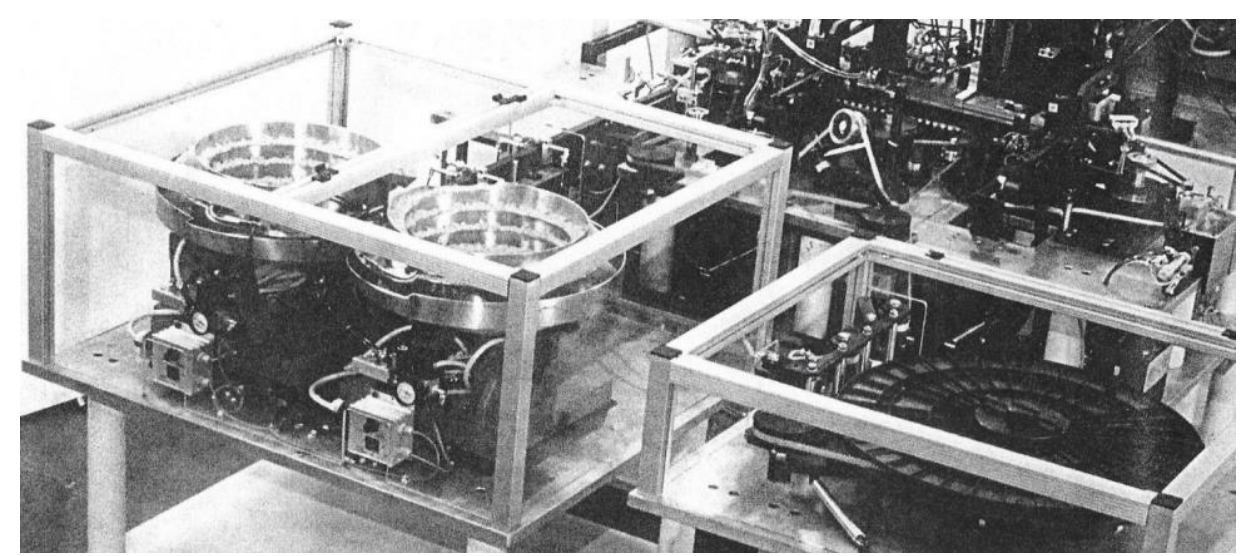

Рисунок 2. Автоматический производственный комплекс для изготовления, сборки и испытаний (контроля качества и работоспособности) контактных

\section{систем связи}

Как видно из рисунка, для штамповки деталей применяется автоматизированная технология штамповки из пружинной ленты, а для металлических деталей, полученных литьём под давлением, и для пластиковых деталей, полученных литьём на термопластавтоматах, применяются специальные бункера, откуда детали по локальным конвейерам поступают на центральный сборочный конвейер системы.

Рассмотрим применение в системах микроавтоматики микродвигателей, высокомоментных и при этом работающих на базе принципов обратного пьезоэлектрического эффекта.

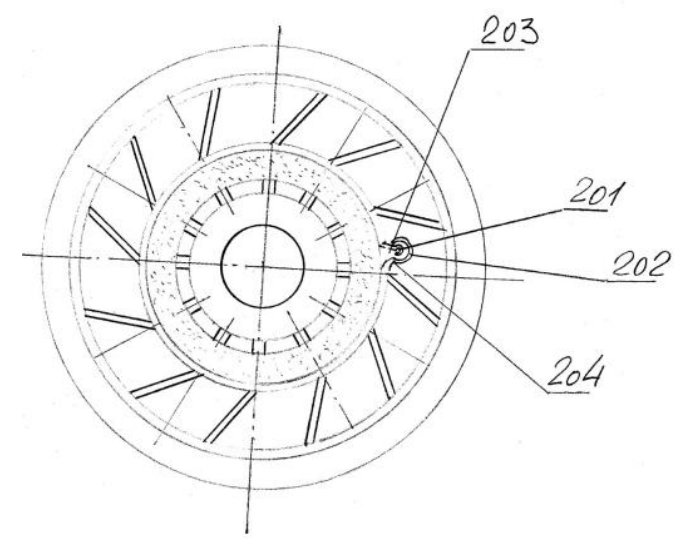

Рисунок 3. Пьезоэлектрический двигатель 
На рисунке 3 показан пьезоэлектрический двигатель в радиальном поперечном сечении. Цифрами на рисунке обозначены:

201. ось замка, фиксирующего упругую ленту с профилированными рабочими лепестками на наружном диаметре пьезокерамического кольца

202. элементы дизайна пружинного замка, фиксирующего и ориентирующего упругую ленту с профилированными рабочими лепестками на наружном диаметре пьезокерамического кольца

203. элементы дизайна пружинного замка, фиксирующего и ориентирующего упругую ленту с профилированными рабочими лепестками на наружном диаметре пьезокерамического кольца

204. элементы дизайна пружинного замка, фиксирующего и ориентирующего упругую ленту с профилированными рабочими лепестками на наружном диаметре пьезокерамического кольца

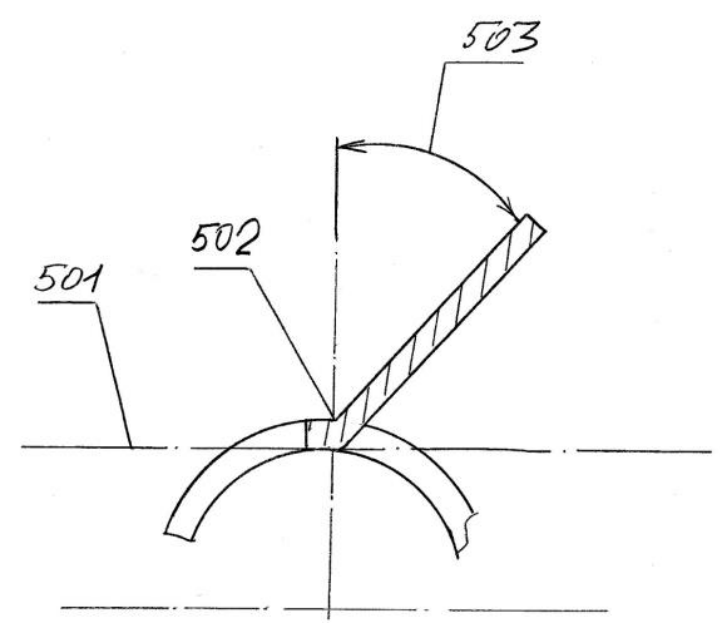

Рисунок 4. Фрагмент формирования рабочего лепестка пружинной ленты двигателя

На рисунке 4 показан фрагмент формирования рабочего лепестка пружинной ленты двигателя. Цифрами на рисунке обозначены:

501. ось поворота плоскости упругого профилированного рабочего лепестка

502. внешний радиус поворота

503. угол изгиба ленты у основания рабочего лепестка по отношению к вертикальной плоскости 


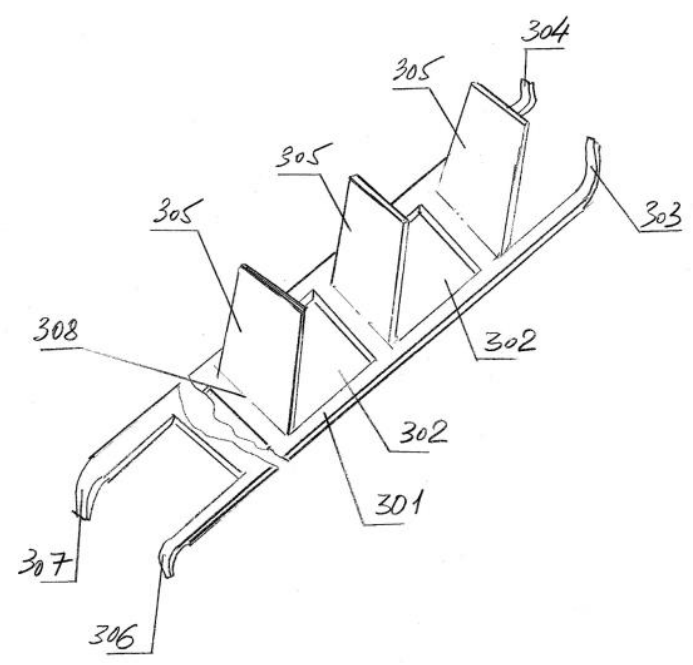

Рисунок 5. Пружинная лента пьезоэлектрического двигателя с рабочими лепестками

На рисунке 5 показана пружинная лента пьезоэлектрического двигателя с рабочими лепестками. Цифрами на рисунке обозначены:

301. пружинная лента, которая обжимается на пьезокерамическом кольце

302. окно в пружинной ленте, оставшееся после вырубки контура рабочих лепестков

303. концы пружинной ленты при сборке на пьезоэлектрическом керамическом кольце формирующие пружинный замок

304. концы пружинной ленты при сборке на пьезоэлектрическом керамическом кольце формирующие пружинный замок

305. рабочие лепестки

306. концы пружинной ленты при сборке на пьезоэлектрическом керамическом кольце формирующие пружинный замок

307. концы пружинной ленты при сборке на пьезоэлектрическом керамическом кольце формирующие пружинный замок

308. внешний радиус поворота рабочего лепестка 


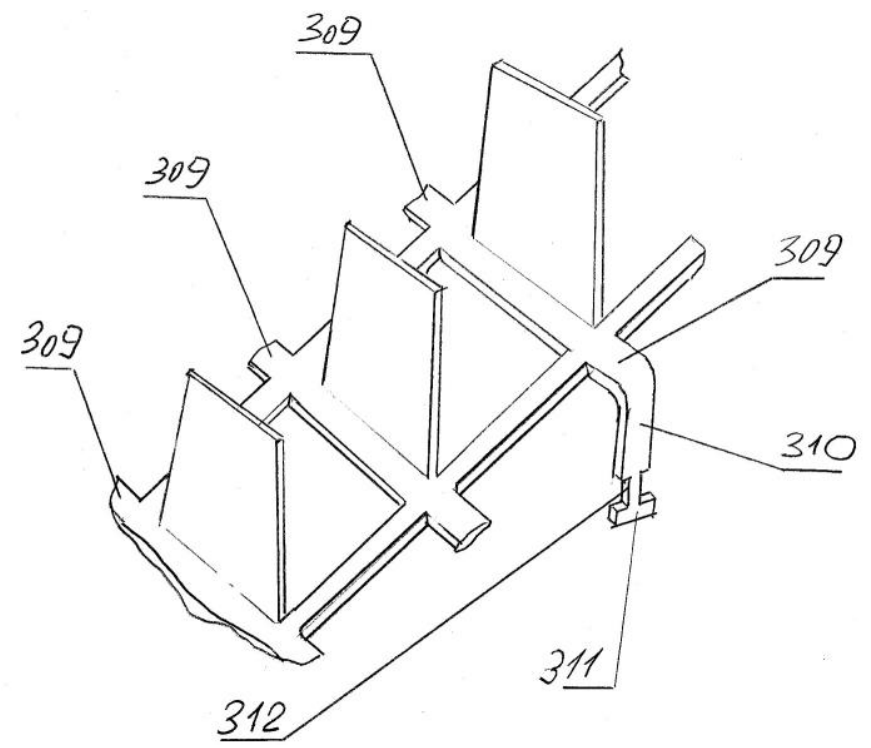

Рисунок 6. Фрагмент пружинной ленты пьезоэлектрического двигателя с рабочими лепестками

На рисунке 6 показан фрагмент пружинной ленты пьезоэлектрического двигателя с рабочими лепестками. Цифрами на рисунке обозначены:

309. дистанционные элементы пружинной ленты

310. элементы пружинного замка

311. элементы пружинного замка

312. элементы пружинного замка

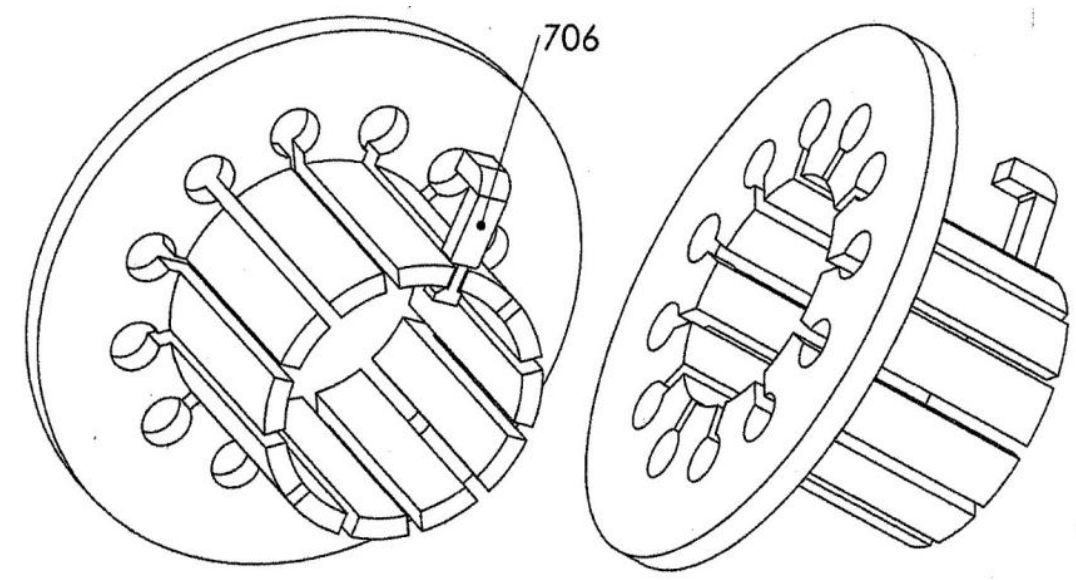

Рисунок 7. Пружинная ось пьезоэлектрического двигателя 


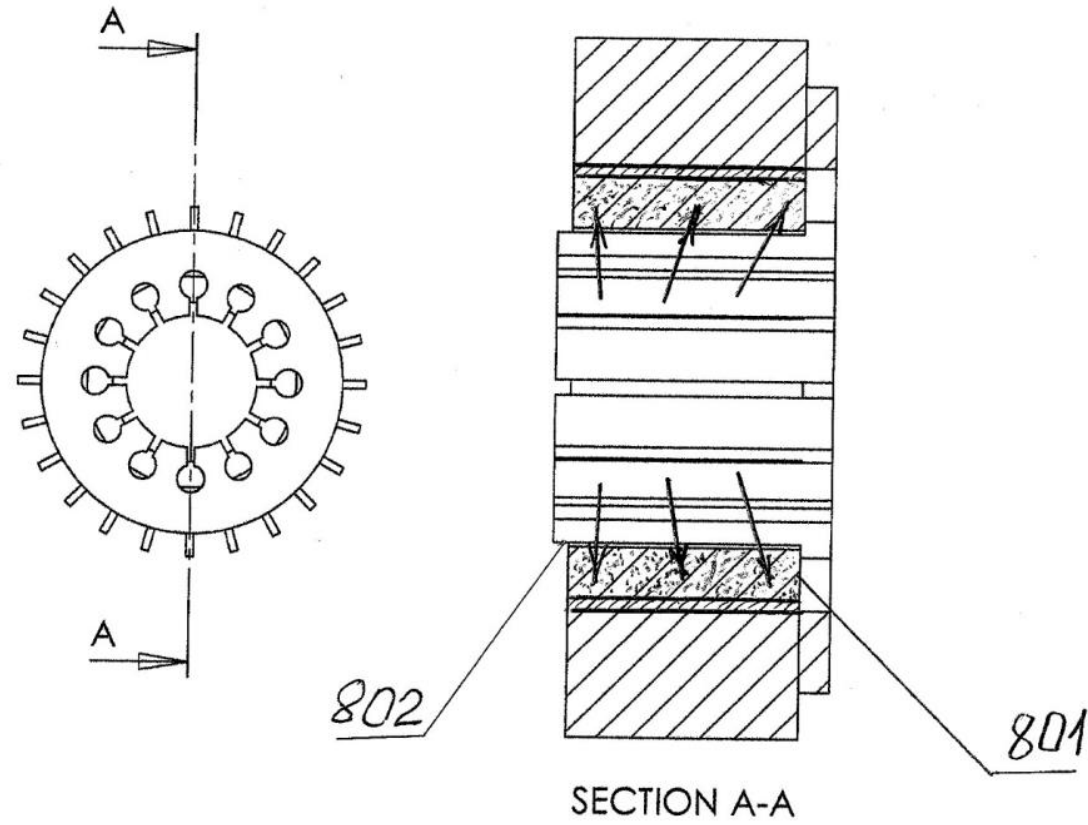

Рисунок 8. Фрагмент пьезоэлектрического двигателя

На рисунке 8 показан Фрагмент пьезоэлектрического двигателя. Цифрами на рисунках обозначены:

706. элементы пружинного замка

801. торец пьезоэлектрического кольца

802. взаимодействие пружинной оси с пьезоэлектрическим кольцом

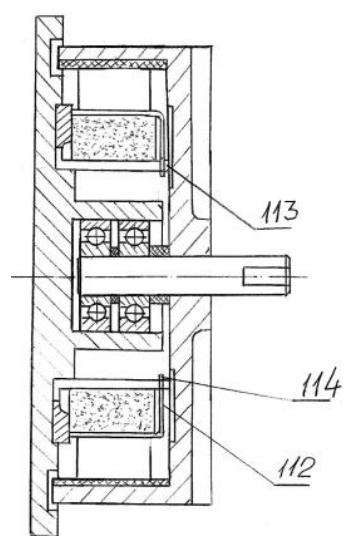

\section{Рисунок 9. Пьезоэлектрический двигатель в осевом сечении}

Цифрами на рисунке обозначены:

112. элементы фиксирующего дистанционного кольца пружинной ленты с рабочими лепестками

113. элементы фиксирующего дистанционного кольца пружинной ленты с рабочими лепестками 
114. элементы фиксирующего дистанционного кольца пружинной ленты с рабочими лепестками

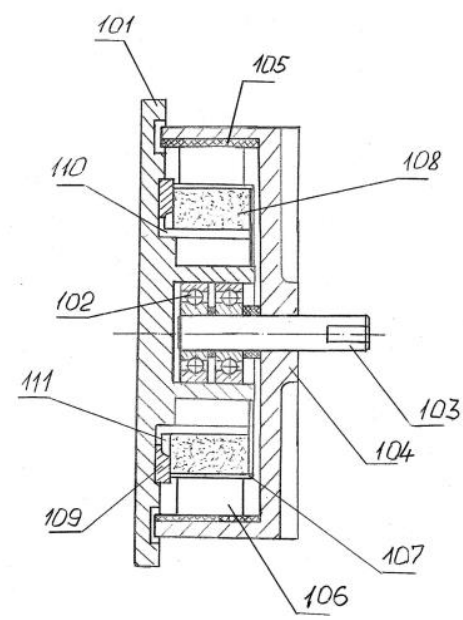

Рисунок 10. Пьезоэлектрический двигатель в осевом сечении

На рисунке 6 показан пьезоэлектрический двигатель в осевом сечении. Цифрами на рисунке обозначены:

101.фланец пьезоэлектрического двигателя

102.центральный подшипник

103. вал пьезоэлектрического двигателя

104.ротор пьезоэлектрического двигателя

105. фрикционное кольцо

106. рабочие лепестки

107.элементы фиксации пружинной ленты с рабочими лепестками на пьезоэлектрическом керамическом кольце

108. пьезоэлектрическое керамическое кольцо

109. опорный дистанционный фланец пружинной оси пьезоэлектрического двигателя

110. пружинная цапфа пружинной оси пьезоэлектрического двигателя

111.базовая канавка - проточка в фланце пружинной оси пьезоэлектрического двигателя 


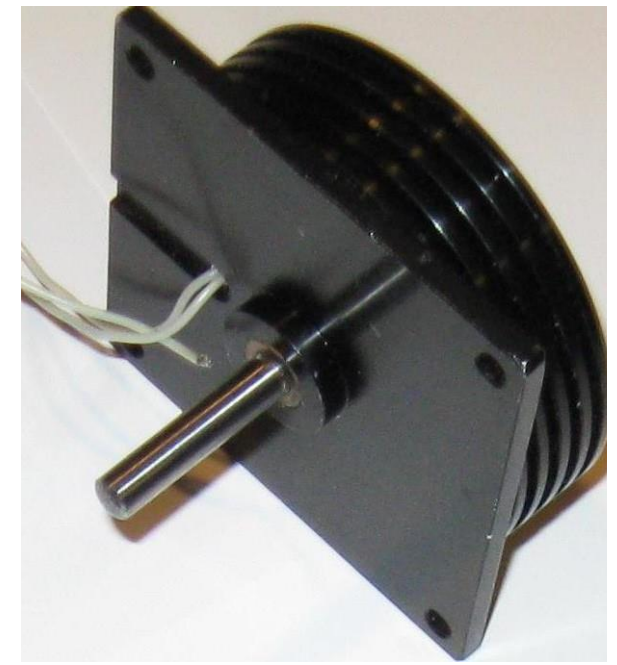

Рисунок 11.

\section{Пьезоэлектрический}

двигатель в сборе

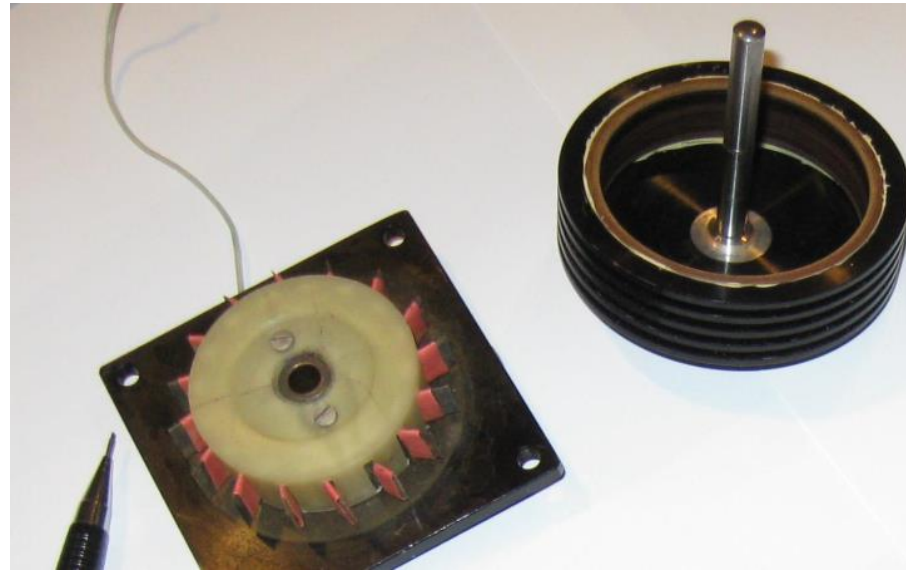

Рисунок 11. Пьезоэлектрический двигатель в разобранном виде и в маситабном факторе

На рисунке 11 показан пьезоэлектрический двигатель в разобранном виде и в масштабном факторе. В правой части рисунка показан ротор пьезоэлектрического двигателя с выходным валом двигателя (запрессован по оси ротора). На корпусе ротора выполнены канавки, играющие роль радиаторов охлаждения и стабилизаторов равномерного вращения ротора под воздействием рабочих лепестков. В корпус ротора запрессовано фрикционное кольцо, через которое рабочие лепестки передают вращающий момент на ротор. В центре рисунка показан установочный и несущий фланец пьезоэлектрического двигателя, на котором закреплена пружинная ось двигателя с пьезоэлектрическим керамическим кольцом с , закреплённым на его пружинной цапфе пьезоэлектрическим кольцом и с пружинной лентой с пружинными лепестками (на рисунке - красного цвета), через фрикционное кольцо находящиеся в упругом фрикционном контакте с ротором двигателя. Такая конструкция двигателя обеспечивает ему и его рабочему методу использования обратного пьезоэлектрического эффекта универсальность, согласно которой двигатель может работать и как двигатель, и как шаговый двигатель.

Переход от одного варианта к другому может осуществляться посредством команд - импульсов от драйвера и не требует никаких модификаций конструктива двигателя. 


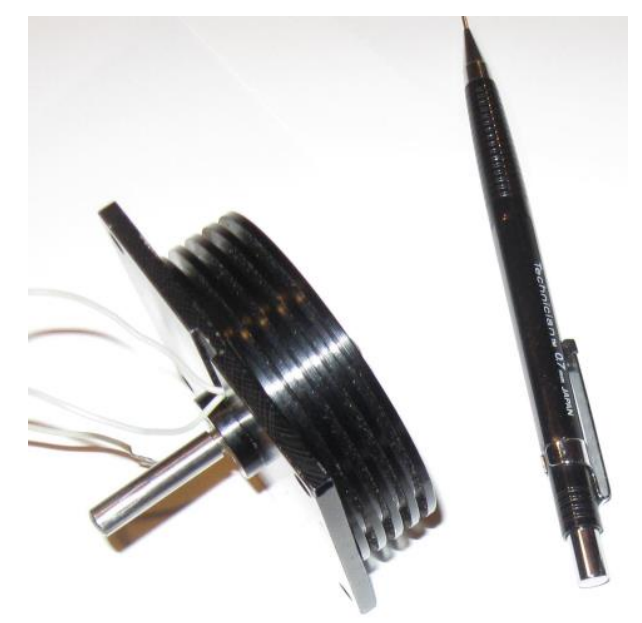

Рисунок 12. Пьезоэлектрический двигатель

На рисунке 12 показан пьезоэлектрический двигатель, работающий по принципу обратного пьезоэлектрического эффекта, в масштабном факторе.

Этот тип двигателя наиболее эффективен в системах автоматики и в автоматических модулях, входящих в гибкие производственные системы, когда необходимы качества шагового двигателя в части точности и стабильности работы, но нет необходимости в электромагнитных компонентах и их производных, создающих множество электронных помех и совершенно не пригодных к работе в условиях применения электромагнитной резонансной спектроскопии.

\section{A list of references, patent and licensing information}

1. Smart absorbent article, components, and process of making [Electronic resource]: pat. US 20190167489 / Hellmold, Jens - Publ. date 06.06.2019. - Mode of access:http://appft.uspto.gov/netacgi/nphParser?Sect1=PTO1\&Sect2=HITOFF\&d=PG01 $\& \mathrm{p}=1 \& \mathrm{u}=\% 2 \mathrm{Fnetahtml} \% 2 \mathrm{FPTO} \% 2 \mathrm{Fsrchnum} . \mathrm{html} \& \mathrm{r}=1 \& \mathrm{f}=\mathrm{G} \& \mathrm{l}=50 \& \mathrm{~s} 1=\% 2220190167$ 489\%22.PGNR.\&OS=DN/20190167489\&RS=DN/20190167489. - Date of access: 18.09.2020.

2. Smart absorbent article, components, and process of making [Electronic resource]: pat. US 20190167489 / Hellmold, Jens - Publ. date 06.06.2019. - Mode of access:http://appft.uspto.gov/netacgi/nphParser?Sect1=PTO1\&Sect2=HITOFF\&d=PG01 $\& \mathrm{p}=1 \& \mathrm{u}=\% 2$ Fnetahtml\%2FPTO $\% 2$ Fsrchnum.html\& $\mathrm{r}=1 \& \mathrm{f}=\mathrm{G} \& \mathrm{l}=50 \& \mathrm{~s} 1=\% 2220190167$ 489\%22.PGNR.\&OS=DN/20190167489\&RS=DN/20190167489. - Date of access: 18.09.2020.

3. Methods and system for managing intellectual property using a blockchain [Electronic resource]: pat. US 20180285996 / Moses T. Ma - Publ. date 04.10.2018. - Mode of access: http://appft.uspto.gov/netacgi/nphParser?Sect1=PTO1 $\&$ Sect $2=$ HITOFF $\& \mathrm{~d}=\mathrm{PG} 01 \& \mathrm{p}=1 \& \mathrm{u}=\% 2$ Fnetahtml $\% 2$ FPTO $\% 2$ Frchnum.html $\& \mathrm{r}=1 \& \mathrm{f}=$ $\mathrm{G} \& \mathrm{l}=50 \& \mathrm{~s} 1=\% 2220180285996 \% 22 . \mathrm{PGNR} . \& O S=\mathrm{DN} / 20180285996 \& \mathrm{RS}=\mathrm{DN} / 20180285$ 996. - Date of access: 18.09.2020 